\title{
Reduced Heart Rate and Cardiac Output Differentially Affect Angiogenesis, Growth, and Development in Early Chicken Embryos (Gallus domesticus)
}

\author{
Sylvia R. Branum* \\ Miho Yamada-Fisher \\ Warren Burggren \\ Developmental Integrative Biology Research Cluster, \\ Department of Biological Sciences, University of North \\ Texas, 1155 Union Circle 305220, Denton, Texas 76203
}

Accepted 3/4/2013; Electronically Published 4/19/2013

\begin{abstract}
An increase in both vascular circumferential tension and shear stress in the developing vasculature of the chicken embryo has been hypothesized to stimulate angiogenesis in the developing peripheral circulation chorioallantoic membrane (CAM). To test this hypothesis, angiogenesis in the CAM, development, and growth were measured in the early chicken embryo, following acute and chronic topical application of the purely bradycardic drug ZD7288. At hour 56, ZD7288 reduced heart rate $\left(f_{\mathrm{H}}\right)$ by $\sim 30 \%$ but had no significant effect on stroke volume $(\sim 0.19 \pm 0.2 \mu \mathrm{L})$, collectively resulting in a significant fall in cardiac output (CO) from $\sim 27 \pm 3$ to $18 \pm 2 \mu \mathrm{L} \mathrm{min}^{-1}$. Mean $f_{\mathrm{H}}$ at $72 \mathrm{~h}$ of development was similarly significantly lowered by acute ZD7288 treatment $(250 \mu \mathrm{M})$ to $128 \pm 0.3$ beats $\mathrm{min}^{-1}$, compared with $174.5 \pm 0.3$ and $174.7 \pm 0.8$ beats min $^{-1}$ in control and Pannett-Compton (P-C) saline-treated embryos, respectively. Chronic dosing with ZD7288-and the attendant decreases in $f_{\mathrm{H}}$ and $\mathrm{CO}$ - did not change eye diameter or cervical flexion (key indicators of development rate) at $120 \mathrm{~h}$ but significantly reduced overall growth (wet and dry body mass decreased by 20\%). CAM vessel density index (reflecting angiogenesis) measured $200-400 \mu \mathrm{m}$ from the umbilical stalk was not altered, but ZD7288 reduced vessel numbers-and therefore vessel density—by 13\%-16\% more distally (500-600 $\mu \mathrm{m}$ from umbilical stalk) in the CAM. In the ZD7288-treated embryos, a decrease in vessel length was found within the second branch order ( $\sim 300-400 \mu \mathrm{m}$ from the umbilical stock), while a decrease in vessel diameter was found closer to the umbilical stock, beginning in the first branch order $(\sim 200-300 \mu \mathrm{m})$. Paradoxically, chronic application of $\mathrm{P}-\mathrm{C}$ saline also reduced pe-
\end{abstract}

* Corresponding author; e-mail: sylviarbranum@gmail.com.

Physiological and Biochemical Zoology 86(3):370-382. 2013. (C) 2013 by The University of Chicago. All rights reserved. 1522-2152/2013/8603-2189\$15.00. DOI: $10.1086 / 670594$ ripheral CAM vessel density index at 500 and $600 \mu \mathrm{m}$ by $13 \%$ and $7 \%$, respectively, likely from washout of local angiogenic factors. In summary, decreased $f_{\mathrm{H}}$ with reduced CO did not slow development rate but reduced embryonic growth rate and angiogenesis in the CAM periphery. This study demonstrates for the first time that different processes in the ontogeny of the early vertebrate embryo (i.e., hypertrophic growth vs. development) have differential sensitivities to altered convective blood flow.

\section{Introduction}

Angiogenesis results from a complex combination of genetically directed morphogenesis and the influence of local environmental factors, both biochemical and mechanical (e.g., Jones et al. 2006; Lee et al. 2009; Adams and Eichmann 2010; Buschmann et al. 2010; Kaunas et al. 2011; Knudsen and Kleinstreuer 2011; Heinke et al. 2012; Burggren 2013). Chief among the mechanical influences are both blood pressure and flow (Isogai et al. 2003; le Noble et al. 2008). Blood pressure creates both an absolute transmural pressure as well as circumferential stretch (tension) in compliant vessels through the fluctuating blood pressure associated with the cardiac cycle. Blood flow stimulates angiogenesis by generating a shear stress on the inner surface of the vessel that parallels the direction of the blood flow. Endothelial cells comprising the inner layer of the vasculature-and those cells lining the sprouting blood vessel tips-respond to such stressors by proliferating under the influence of vascular endothelial growth factor (VEGF) and other paracrine secretions. New vessels and the eventual throughflow of blood occur as a result (see reviews in Groenendijk et al. 2007; le Noble et al. 2008; Egginton 2011). Arterial (as opposed to venous) identity then arises from the greater pulsatility and shear stresses that eventually emerge in the arterial vasculature (Buschmann et al. 2010). Angiogenesis is crucial to the completion of the capillary network and the connecting of developing arteries and veins. Indeed, failure of angiogenesis in early embryonic development has long been known to lead to rapid embryonic death, as evident from observations of VEGF knockout mice that fail to develop a complete microvasculature (Carmeliet et al. 1996; Ferrara et al. 1996).

While well documented in mature vertebrates, the dependence of early embryonic angiogenesis on the onset of heart beat, blood pressure generation, and blood flow has not been 
well established in the embryo. A complicating factor is that blood pressure and flow are not an absolute requirement for organogenesis and growth in the early vertebrate embryo, because development in early embryos will continue for some time when convective blood flow and mass transport are experimentally eliminated (Mellish et al. 1994; Pelster and Burggren 1996; Burggren et al. 2000, 2004). This independence of development and growth from convective blood flow occurs because diffusion provides adequate mass transport during early embryo development. Eventually, a combination of escalating gas exchange and nutrient demands, coupled with increasing diffusion distances of the growing embryo, necessitates the development of an active internal convective blood flow (for review, see Burggren 2004).

Onset of blood pressure and flow generation-a metabolically expensive process-occurs well in advance of the embryo's absolute need for material transport, a phenomenon that has been termed "prosynchronotropy" (Territo and Burggren 1998). Unanswered is the question of why cardiac contraction and blood convection occur earlier than actually required for bulk transport. An as yet untested hypothesis is that the initial beating of the heart is not for material transport but rather to provide the pulsatile pressure and flow-and the attendant circumferential stretch and shear stresses-in the peripheral circulation as a necessary stimulant for angiogenesis in the early embryo (Burggren 2004). The hypothesis of prosynchronotropy has recently been refined to that of synangiotropy, namely, that blood pressure and flow appear just in time for the need for angiotropy rather than bulk transport (Burggren 2013). To test this hypothesis, this study used the purely bradycardic drug ZD7288 to induce chronic bradycardia in the chicken embryo from 72 to $120 \mathrm{~h}$ of development. Bradycardia increases pulse pressure in the arterial vascular beds as a result of the lengthened period of diastolic pressure runoff, thus potentially increasing vascular circumferential stress. If bradycardia induces a decreased cardiac output (CO), it will additionally reduce shear stress on the vascular endothelial lining.

In early stages of avian development, bradycardia is not accompanied by a compensatory increase in stroke volume, and so a reduction in $\mathrm{CO}$ accompanies heart rate reduction (Bowers et al. 1996; W. Burggren and M. Yamada-Fisher, unpublished manuscript). Using experimentally induced bradycardia as a tool for lower CO, we have also assessed rates of embryonic development, growth, and angiogenesis in both control populations and those chronically exposed to either PannettCompton (P-C) saline or ZD7288.

\section{Material and Methods}

\section{Source of Experimental Animals}

Fertilized eggs of the white leghorn chicken (Gallus domesticus L.) were obtained from Texas A\&M University (College Station, TX) and shipped to the University of North Texas (Denton, TX). Eggs were incubated in a Hova-Bator Styrofoam incubator at a constant temperature of $37.5^{\circ} \pm 0.5^{\circ} \mathrm{C}$ and $55 \%-60 \%$ hu- midity. After $48 \mathrm{~h}$ of incubation, the eggs were removed from the incubator and transferred into a shell-less culture system.

\section{Shell-Less Culture}

To facilitate drug delivery and measurements, shell-less embryo cultures were prepared after the method of Hamamichi and Nishigori (2001). This method enables growth of the embryo for up to $5 \mathrm{~d}$, while allowing full access to the embryo and its vasculature for manipulation and observation (fig. 1). To prepare a shell-less culture, each incubated egg was removed from the incubator and positioned horizontally at room temperature on an egg crate for $10 \mathrm{~min}$ to allow the embryo to be positioned correctly for harvesting. The egg surface was then lightly sprayed with $70 \%$ ethanol to reduce microbial contamination from the egg surface and then allowed to air-dry. Each egg was cracked, and the whole egg contents were transferred into an autoclaved Kimax crystallizing dish $(60 \mathrm{~mm} \times 35 \mathrm{~mm})$ under aseptic conditions. Any air bubbles surrounding the yolk were removed with a sterile Kimwipe. The dish was covered with clear polyethylene film secured with an elastic rubber band. Cultured embryos were then numbered and reincubated at $37.5^{\circ} \pm 0.5^{\circ} \mathrm{C}$ for further development and use. Care was taken to include only cultures with intact yolks that had the blastodisc positioned on the uppermost side of the yolk.

\section{Experimental Design and Embryo Populations}

Three populations of shell-less culture embryos were created. The control embryos were untreated in any way and were simply grown in the culture conditions described above. P-C saline-treated embryos grown in culture received topical application of P-C saline $\left(2.2 \mathrm{~mol} \mathrm{~L}^{-1} \mathrm{NaCl}, 0.21 \mathrm{~mol} \mathrm{~L}^{-1} \mathrm{KCl}\right.$, $\mathrm{pH}=7$; after Stern and Holland 1993) to determine any mechanical or physiological effects of fluid placement on the embryo. The $\mathrm{Po}_{2}$ of the culture medium, which was air saturated, was $\sim 150 \mathrm{mmHg}$. Finally, a group of embryos, grown in culture as described above, received topical application of the bradycardic agent ZD7288. This drug blocks the $I_{\mathrm{f}}$ cardiac pacemaker channels in the heart pacemaker, reducing heart rate without creating an inotropic cardiac effect (Yusuf and Camm 2003; Luo et al. 2006).

\section{Drug Solutions and Delivery Methods}

ZD7288 was obtained from Tocris Bioscience (Ellisville, MO). A stock solution of $10 \mathrm{mg}$ ZD7288 in $1 \mathrm{~mL}$ of autoclaved distilled water was made and stored at $-20^{\circ} \mathrm{C}$. Subsequent solutions of specific concentrations were made using P-C saline (Pannett and Compton 1924), which was originally developed as a superior medium to standard chick Ringer solutions for growing blastodiscs in vitro.

The two-dimensional nature of shell-less culture was effective in allowing an even distribution of solution and drug to the embryo and its extraembryonic tissues. Preliminary observations using P-C saline solution tinted with Evans blue dye showed that 


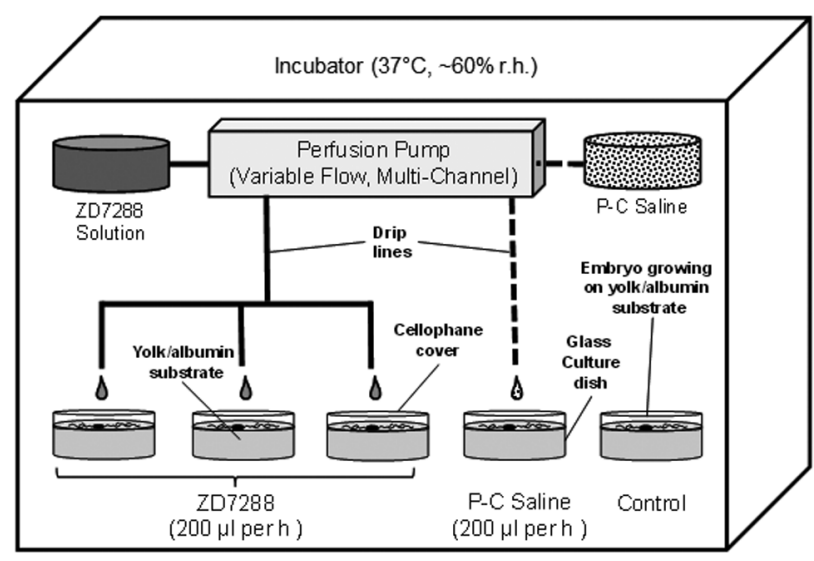

Figure 1. Highly schematic diagram of the apparatus used for chronic infusion of either ZD7288 solution or Pannett-Compton (P-C) saline (control).

topical application of a $5-\mu \mathrm{L}$ drop of solution directly above the embryo's body wall resulted in broad, even distribution of the solution across both the embryo and the surrounding chorioallantoic membrane (CAM). At the same time, excess solution ran off the surface of the embryo and collected remotely at the far edges of the culture dish, thus preventing accumulation of fluid over the embryo itself (fig. 1).

In acute experiments, the dish containing a cultured embryo was briefly removed from the incubator. A single predetermined dose of ZD7288 solution was topically applied from a sterilized transfer pipette inserted through the polyethylene wrap cover. Acute experiments were carried out on embryos of the following ages: 55-56, 72, 96, and $120 \mathrm{~h}$.

For chronic dosing, three dishes with 48-h embryos were placed in a Lyon Reptilife incubator (model RL1\&2) set at $37^{\circ} \pm 0.5^{\circ} \mathrm{C}$ and $55 \%-60 \%$ humidity (fig. 1). A syringe pump (model 352; Sage Instruments, Boston, MA) was also placed within the incubator to ensure precise temperature control of drug or saline delivery. Three Bristoline $10-\mathrm{cm}^{3}$ glass syringes containing ZD7288 or P-C saline were secured on the syringe pump and used for continuous drug delivery to three embryos simultaneously. The tip of a 10-mm-long PE 100 tubing was glued into the tip of a 50-mm-long PE 240 polyethylene tube and attached by a plastic on/off connector to each syringe and then was placed through the cellophane wrap directly over each embryo's heart. This chronic drug delivery system slowly flushed each embryo's surface with P-C saline or ZD7288 solution at a rate of $200 \mu \mathrm{L} \mathrm{h}^{-1}$ for a 24 -h exposure period, for a total of $4.80 \mathrm{~mL}$ of delivered solution by age $72 \mathrm{~h}$. Control embryos were cultured within the incubator at the same temperature and for the same time period but did not receive PC saline or ZD7288.

\section{Treatment and Heart Measurement Protocols}

Each of the lids of the incubators, in which the acute populations were housed, had a transparent section through which the beating heart of the cultured embryo could be visually observed. Heart rate $\left(f_{\mathrm{H}}\right)$ in embryos from P-C saline-treated and ZD7288 groups was measured by counting the number of heart beats in two successive 15-s intervals separated by a 10$s$ period and transformed to beats per minute. After this initial heart rate measurement, embryos in their culture dish were then briefly removed from the incubator. In one acute population, a single $5-\mu \mathrm{L}$ aliquot of $\mathrm{P}-\mathrm{C}$ saline was applied topically to the embryo. In the experimental population, bradycardia was induced by a single $5-\mu \mathrm{L}$ aliquot of one of the range of concentrations $(30,250$, and $1,000 \mu \mathrm{M})$ of topically applied ZD7288. The control population was left untreated. Embryos were then immediately placed back into the incubator. Heart rate continued to be taken every 10 min after administration of the drug for $\sim 10 \mathrm{~h}$. This protocol was carried out at 56, 72, 96 , and $120 \mathrm{~h}$ of development.

For chronic treatments, embryos were dosed with 7.5, 10, 15, 20, and $30 \mu \mathrm{M}$ ZD7288 for $24 \mathrm{~h}$, starting at $48 \mathrm{~h}$ and continuing through $72 \mathrm{~h}$ of development. In preliminary experiments used to refine the protocol, chronic treatment of 30 $\mu \mathrm{M}$ ZD7288 at a rate of $200 \mu \mathrm{L} \mathrm{h}^{-1}$ (about $405-\mu \mathrm{L}$ drops $\mathrm{h}^{-1}$ ) achieved the same degree of bradycardia as the maximum acute bradycardic response produced by a single drop of $5 \mu \mathrm{L}$ of 500 $\mu \mathrm{M}$ ZD7288. The subsequent maximum chronic concentration used was thus adapted from these results.

\section{Blood Velocity and Flow Measurements}

Blood flow measurements were made in shell-less culture embryos from 56 to $60 \mathrm{~h}$ of development. This stage of development is $\sim 10 \mathrm{~h}$ younger than the stage at which some of the other measurements were made but was also the stage yielding the most precise signals with the lowest variation. Dorsal aortic blood velocity was measured with a $20-\mathrm{MHz}$ pulsed Doppler flowmeter and a $0.5-\mathrm{mm}$-diameter piezoelectric crystal (Iowa Doppler Products, Iowa City, IA) positioned over the dorsal aorta at a $45^{\circ}$ angle. Analog signals were logged onto an eightchannel PowerLab III data acquisition system (ADInstruments, Colorado Springs, CO). Mean aortic velocity and $f_{\mathrm{H}}$ were calculated by the data acquisition software. The diameter of the dorsal aorta was measured with a filar micrometer eyepiece. From these measures, stroke volume $(\mu \mathrm{L})$ and $\mathrm{CO}(\mu \mathrm{L} / \mathrm{min})$ were calculated. (Note that dorsal aortic blood flow measured at this point does not include $\mathrm{CO}$ going to the developing embryonic head [Hu and Clark 1989].)

\section{CAM Vessel Density Index Measurement}

CAM vessel density index protocols were created using previously published conventions (Strick et al. 1991; Höper and Jahn 1995; Corona et al. 1999). Images of each embryo were taken at $\times 3.5$ using a Javelin camera mounted on a Leica WILD M3Z microscope. To measure the CAM vessel density index of the extraembryonic vasculature, Image Pro software was used to draw five concentric circles $(200,300,400,500$, and 600 $\mu \mathrm{m})$ centered directly over the umbilical stock of the embryo 
(fig. 2). CAM vessel density index was then calculated by counting the number of clearly visible vessels that intersected a given circle around its entire circumference. An index for each location was counted separately, starting with the $200-\mu \mathrm{m}$ circle and moving outward to $600 \mu \mathrm{m}$.

\section{Vessel Branch Order, Length, and Diameter Measurements}

Images taken to assess the CAM vessel density index were also used to measure number of vessels per branch order, vessel length, and vessel diameter. Branch order was quantified as follows: first order being the main umbilical vessel extending from the embryo on both sides, second order being the vessels branching off the first order vessel, and so forth. Both left and right sides of the embryo were measured for statistical comparison.

The number of vessels per branch order was measured to the fourth branch order, beyond which visual quantification became imprecise. Vessel length was measured from the beginning of the vessel to the end of the vessel, where it branches off into the next order. Measurements were repeated and then averaged. Vessel diameter was measured at $25 \%$ and $75 \%$ of the length of the vessel to ensure that representative measurements of the vessel were obtained. Both vessel length and vessel diameter were measured out to the third branch order.

\section{Eye Diameter/Cervical Flexure Measurement}

Eye diameter $(\mu \mathrm{m})$ and cervical flexure $\left(^{\circ}\right)$ are conventional measures of development during early developmental stages. In the current experiments, these variables were assessed with Image-Pro software (ver. 4.1). Calibrated images taken with a dissecting microscope and camera at a total magnification of $\times 16$ were used for measurement.

\section{Embryo Dry and Wet Mass Measurements}

After the vasculature measurements, embryos were removed from culture to acquire wet and dry mass. The embryo was carefully cut away from the surrounding vessels, and extraembryonic membranes were pulled off of the embryo with forceps, blotted with filter paper to absorb excess liquid, and placed into a preweighed plastic dish for wet mass measurement. The dish containing the embryo was then placed into a drying oven at $80^{\circ} \mathrm{C}$ for $48 \mathrm{~h}$ and then weighed to obtain dry mass.

\section{Statistical Analyses}

Effects of development and chronic exposure to ZD7288 on heart rate, eye diameter, cervical flexure, and wet and dry mass were assessed using one-way ANOVA, followed by Tukey post hoc tests to determine pairwise differences. Two-way ANOVA was used to assess statistical differences in CAM vessel density index caused by chronic exposure to ZD7288 and acute heart rate caused by acute exposure to ZD7288 and development, respectively. Two-way ANOVA was also used to assess the dif-

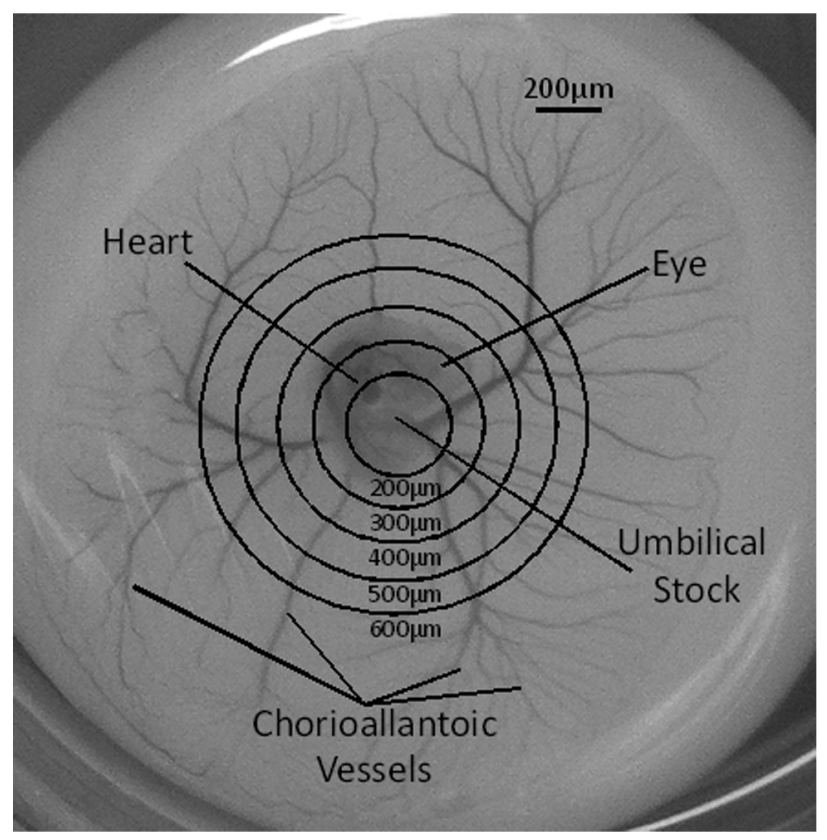

Figure 2. Technique for measuring chorioallantoic membrane (CAM) vessel density index using concentric circles at $100-\mu \mathrm{m}$ intervals, all centering around the root of the umbilical stock. CAM vessel density index was calculated by counting the number of clearly visible vessels that intersected each circle around its entire circumference. A color version of this figure is available in the online edition of Physiological and Biochemical Zoology.

ference between the number of vessels per order, vessel length, and vessel diameter. Where significant differences were found, Tukey post hoc tests were used for pairwise comparisons. We used $t$-tests to determine whether there was a difference between the number of vessels on the right and the left side of embryo. All variables are represented as mean $\pm 1 \mathrm{SE}$, with $\alpha=0.05$. All statistical analyses were performed using SigmaStat and SigmaPlot software.

\section{Results}

\section{Heart Rate and Blood Flow in Control and Saline-Treated Populations}

Mean $f_{\mathrm{H}}$ at 56 h of development in control embryos was $\sim 130$ 140 beats $\mathrm{min}^{-1}$, rising to $\sim 170$ beats $\min ^{-1}$ by $72 \mathrm{~h}$ of development (fig. 3). As expected from prior studies, $f_{\mathrm{H}}$ began to plateau at $\sim 220$ beats $\min ^{-1}$ at $96 \mathrm{~h}$ and increased only slightly to 230 beats $\min ^{-1}$ at $120 \mathrm{~h}$ (fig. 3).

Blood velocity in the dorsal aorta at $56 \mathrm{~h}$ ranged from 18 to $22 \mathrm{~mm} \mathrm{~s}^{-1}$ at peak systolic ejection, falling back to 0 during diastole (fig. 4), indicating little or no windkessel effect that would otherwise maintain substantial aortic blood flow during diastole.

Stroke volume at $56 \mathrm{~h}$ in saline-treated embryos was approximately $0.19 \mu \mathrm{L}$, leading to a $\mathrm{CO}$ of $\sim 27 \mu \mathrm{L} \min ^{-1}$ at $f_{\mathrm{H}}$ of 140 beats $\mathrm{min}^{-1}$ in control populations (fig. 5). Acute topical 


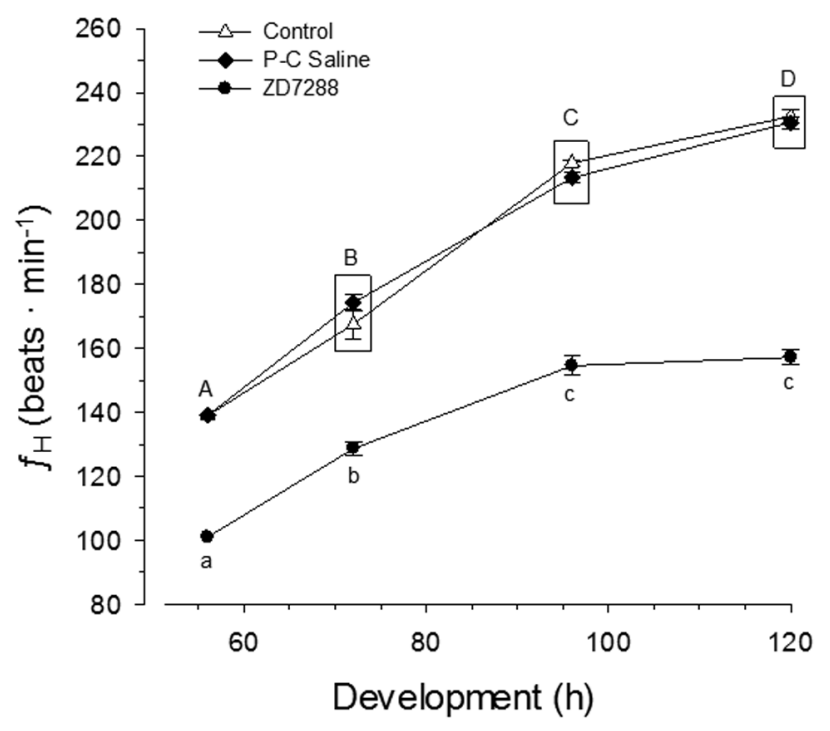

Figure 3. Heart rate $\left(f_{\mathrm{H}}\right)$ as a function of development in control embryos and those acutely treated with either Pannett-Compton (PC) saline or $5 \mu \mathrm{L}$ of $500 \mu \mathrm{M}$ ZD7288. Means \pm SE are plotted; $n=6$ for each group. Boxes enclose statistically identical means. Letters indicate differences in heart rate over the course of development. Uppercase letters show differences in control and P-C saline groups, while lowercase letters show differences between ZD7288 groups.

application of P-C saline had no significant effect $(P<0.05)$ on any measured cardiovascular variable.

\section{Acute Cardiovascular Effects of ZD7288}

Initial experiments were conducted to determine the effective dose ranges for ZD7288-induced bradycardia in 56-h chicken embryos. $f_{\mathrm{H}}$, expressed as a percentage of control values, decreased significantly with as little as $30 \mu \mathrm{M}$ (fig. 6). The most pronounced effect occurred at $500 \mu \mathrm{M}$, with no further significant drop in $f_{\mathrm{H}}$ at concentrations as high as $1,000 \mu \mathrm{M}$. Subsequent experiments were thus limited to doses in the 30$500 \mu \mathrm{M}$ range.

The time course of ZD7288 effects was then established, along with the effects of the carrier, P-C saline. The dose- and time-dependent effects of acute exposure to three different concentrations of ZD7288 and P-C saline in 72-h embryos are indicated in figure 7. $f_{\mathrm{H}}$ of embryos acutely treated with ZD7288 typically decreased significantly $(P<0.01)$ within $30 \mathrm{~min}$ of application at doses 30,250 , and $500 \mu \mathrm{M}$, with the largest effects occurring at $1-2 \mathrm{~h}$ following exposure but lasting at least $4-5$ h (fig. 7).

$f_{\mathrm{H}}$ at 56 and $72 \mathrm{~h}$ decreased from $\sim 140$ to slightly more than 100 beats $\min ^{-1}$ and from 170 to $\sim 140$ beats $\mathrm{min}^{-1}$, respectively, following acute topical application of ZD7288 (figs. 5A, 7). Stroke volume at $56 \mathrm{~h}$ did not change significantly $(P>0.05)$ following an acute application of ZD7288 and the attendant longer period of diastolic filling accompanying bradycardia (fig. $5 B$ ). As a consequence of a fall in $f_{\mathrm{H}}$ without a compensatory increase in stroke volume in embryos acutely exposed to ZD7288, CO decreased significantly $(P<0.01)$ from $\sim 27$ to 18 $\mu \mathrm{L} \min ^{-1}$ (fig. 5C).

Mean $f_{\mathrm{H}}$ in control and P-C saline-treated (acute) embryos at $72 \mathrm{~h}$ of development were statistically identical at $175 \pm$ 0.3 and $175 \pm 1$ beats $\min ^{-1}(P>0.05)$, respectively (fig. 7 ). Acute dosing with $5 \mu \mathrm{L}$ of all three concentrations of ZD7288 produced a significant decrease from control $f_{\mathrm{H}}$ values within 15 min of drug delivery (fig. 7). Maximum effects were evident within 45-75 min of treatment. Thirty microns of ZD7288 produced an $f_{\mathrm{H}}$ of $\sim 102$ beats $\mathrm{min}^{-1}$ that was significantly lower than the control and P-C saline groups $(P<0.001)$ but significantly higher than the more profoundly reduced $f_{\mathrm{H}}$ of the 250 and $500 \mu \mathrm{M}$ ZD7288 groups $(P<0.001)$, which were not significantly different from each other (one-way ANOVA, $P=$ 0.830; fig. 7).

Acute ZD7288 treatment $(500 \mu \mathrm{M})$ continued to create a significant $(P<0.001)$ bradycardia at both 96 and $120 \mathrm{~h}$ of development. This effect was unrelated to delivery vehicle, since treatment with $\mathrm{P}-\mathrm{C}$ saline caused no significant change in $f_{\mathrm{H}}$ from control at these stages.

\section{Hemodynamic Effects of Chronic Treatment with ZD7288}

Heart rates at $72 \mathrm{~h}$ of development in control embryos (169 \pm 3 beats $\min ^{-1}$ ) and those chronically treated with P-C saline from 48 to $72 \mathrm{~h}\left(172 \pm 3\right.$ beats $\left.\mathrm{min}^{-1}\right)$ were not significantly different $(P>0.05)$. Chronic treatment with 7.5, 10, 15, 20 , and $30 \mu \mathrm{M}$ ZD7288 all created a bradycardia that was significantly different from both the control and the $\mathrm{P}-\mathrm{C}$ saline groups $(P<0.001)$ yet not significantly different from each other $(P>0.05$; fig. 8). Chronic dosing of ZD7288 decreased $f_{\mathrm{H}}$ by $\sim 33 \%$ from $171 \pm 3$ beats $\min ^{-1}$ to an average for all ZD7288 doses of $116 \pm 3$ beats $\min ^{-1}$ at $72 \mathrm{~h}$ of development. The higher doses were accompanied by increased embryo mor-

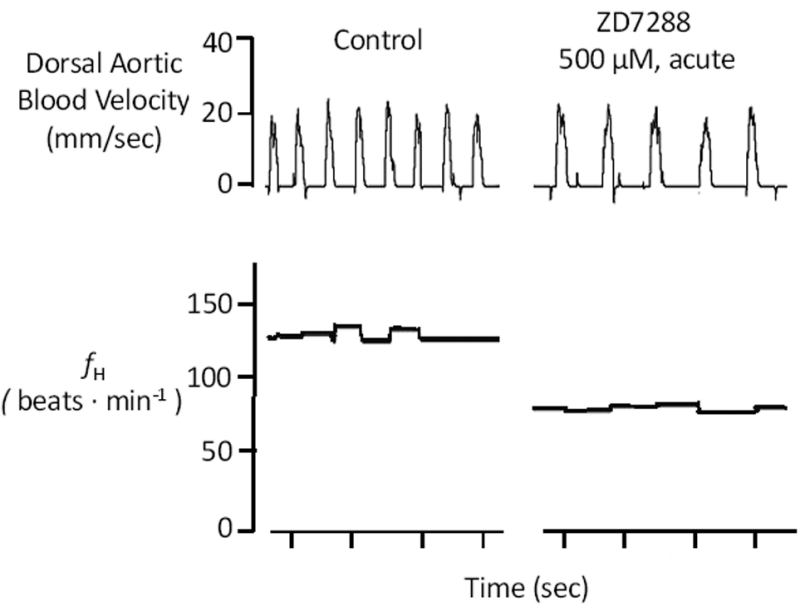

Figure 4. Representative traces of dorsal aortic blood velocity and derived heart rate measured in 56-h chicken embryos before and after acute topical application of the bradycardic agent ZD7288. 

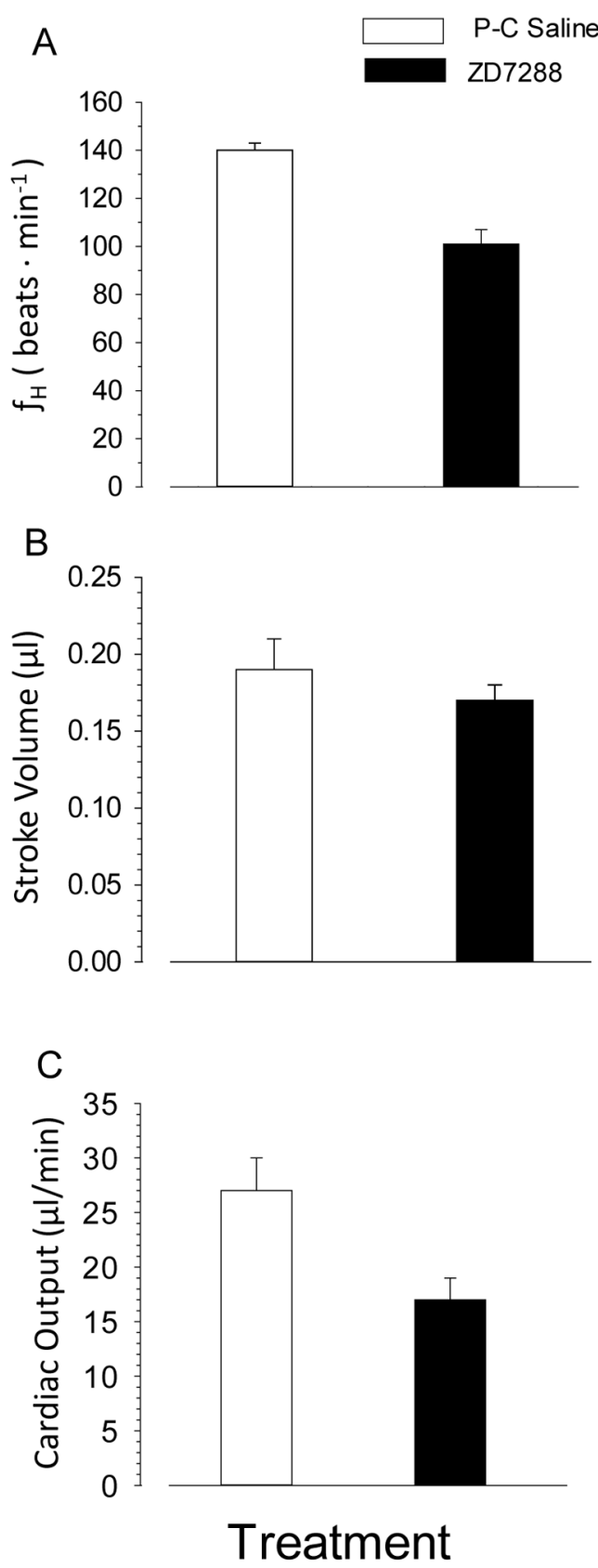

Figure 5. Acute effects of ZD7288 on heart rate $(A)$, stroke volume $(B)$, and cardiac output $(C)$ in 56-h embryos. Note that acute ZD7288 treatment reduces heart rate, but there is no compensatory increase in stroke volume, resulting in a net decrease in cardiac output. Means $\pm 1 \mathrm{SE}$ are plotted; $n=6$ for each group. P-C, Pannett-Compton.

tality, with the lethal chronic dose (defined by the $\mathrm{LD}_{50}$ ) being $30 \mu \mathrm{M}$ ZD7288, since $50 \%$ of the embryos did not survive 24 $\mathrm{h}$ during application of ZD7288.

\section{CAM Vessel Density Index and ZD7288 Treatment}

There was no significant effect of the various ZD7288 concentrations on $f_{\mathrm{H}}$ in chronic experiments (i.e., they were statistically identical populations). Consequently, the CAM vessel density index data for all embryos chronically dosed with the various doses of ZD7288 were merged together to form a total of three groups for statistical analysis: control, P-C saline-treated, and ZD7288-treated groups. CAM vessel density index of control embryos at a distance of 200,300 , and $400 \mu \mathrm{m}$ from the umbilical stalk was $3.2 \pm 0.1,6.2 \pm 0.33$, and $9.5 \pm 0.44$ intersects $\mu \mathrm{m}^{-1}$, respectively (fig. 9).

There was no significant difference in CAM vessel density index between control and treatment groups $(P>0.05)$ proximally at 200,300, and $400 \mu \mathrm{m}$ from the embryo umbilical stalk (fig. 9). However, chronic bradycardia from the application of ZD7288 induced a significant decrease in CAM vessel density index at a distance of $500 \mu \mathrm{m}$ from the umbilical stalk, down to $12.2 \pm 400$ intersects $\mu \mathrm{m}^{-1}$ from a control value of $14.1 \pm 700$ intersects $\mu \mathrm{m}^{-1}(P<0.001)$. Surprisingly, chronic application of $\mathrm{P}-\mathrm{C}$ saline also induced a significant decrease in CAM vessel density index at $500 \mu \mathrm{m}$, down to $12.5 \pm 400$ intersects $\mu \mathrm{m}^{-1}$ compared with the control, $14.3 \pm 700$ intersects $\mu \mathrm{m}^{-1}(P=0.020)$.

At a distance of $600 \mu \mathrm{m}$ from the umbilical stock, there was also a significant decrease $(P<0.001)$ in the CAM vessel density index of both the ZD7288 (16.6 \pm 600 intersects $\left.\mu \mathrm{m}^{-1}\right)$ and the P-C saline $\left(18.5 \pm 600\right.$ intersects $\left.\mu \mathrm{m}^{-1}\right)$ embryos as compared with the control group $\left(19.7 \pm 700\right.$ intersects $\mu \mathrm{m}^{-1}$; fig. 9).

\section{Vessel Number per Branch Order and ZD7288 Treatment}

To quantify the number of blood vessels for each branching order, both sides of the embryos were measured. In the control embryos, there was no significant difference between the number of vessels on the left and the right side of the embryos $(P=0.934)$. Therefore, the left side of the embryo was used for treatment comparisons and statistical analysis. Vessel num-

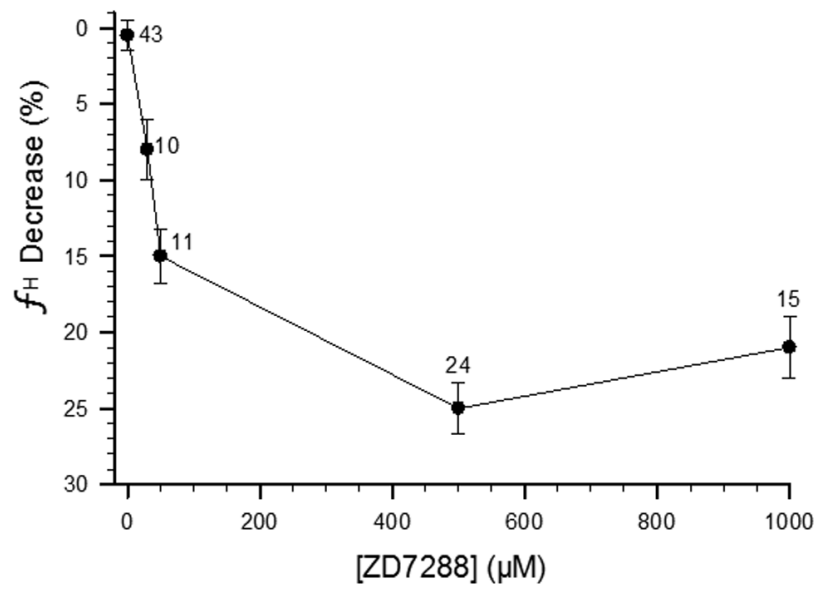

Figure 6. Dose response curve showing percent decrease of heart rate for acute dosing of different concentrations of ZD7288 (ranging from 30 to $1,000 \mu \mathrm{m}$ ) on $56-\mathrm{h}$ embryos. Numbers indicate $n$ values for each measurement. 


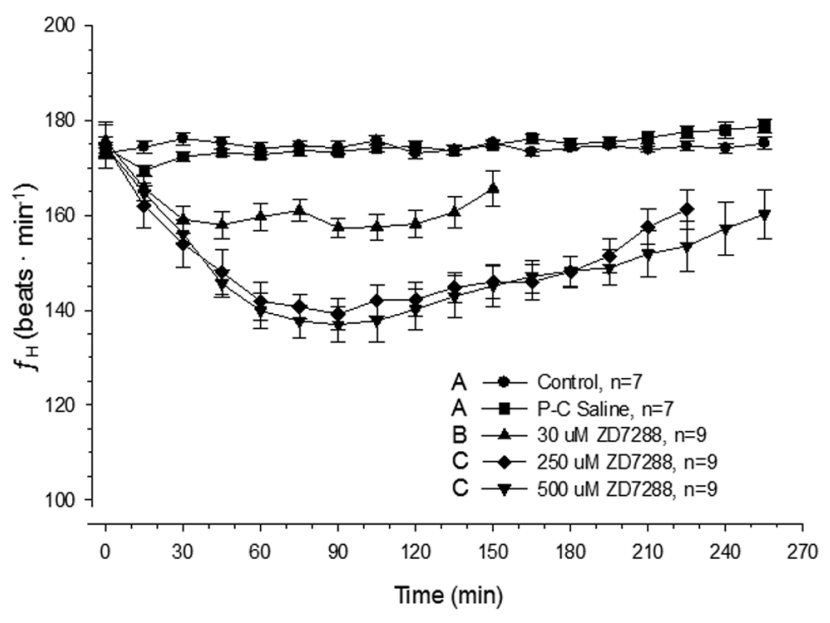

Figure 7. Heart rate at $72 \mathrm{~h}$ of development as a function of control, Pannett-Compton (P-C) saline treatment, and acute ZD7288 dosing at three concentrations. Each point is the mean \pm SE of a 15 -min period. Letters indicate statistical difference between groups, starting at $20 \mathrm{~min}$ after dosing; $n$ values are shown for each condition.

ber per branch order was not significantly different between the first and second branch order for any of the treatments. In the third- and fourth-order vessels within the P-C saline- and ZD7288-treated embryos, the vessel number decreased significantly from control embryos (two-way ANOVA, Tukey pairwise comparison, $P<0.05$; fig. $10 A)$.

\section{Vessel Length and Diameter and ZD7288 Treatment}

Vessel length in the first branch order was not significantly different within treatment groups. In the second order, however, ZD7288-treated embryos showed a significant $40 \%$ decrease from both the control and the P-C saline embryos (fig. 10B). In the third branch order, the P-C saline- and ZD7288-treated embryos both showed a significant $28 \%$ decrease in vessel length from control embryos (two-way ANOVA, Tukey pairwise comparison, $P<0.05$; fig. $10 B)$.

Not surprisingly, as branch order increased, vessel diameter decreased (fig. 10C). Within the first and second branch orders, there was a significant 19\%-14\% decrease, respectively, of vessel diameter in ZD7288-treated embryos from P-C saline and control embryos (two-way ANOVA, Tukey pairwise comparison, $P<0.05)$. Vessel diameter in the third branch order was not significantly different between any of the groups (fig. 10C).

\section{Developmental Effects of Chronic ZD7288 Treatment}

Eye diameter at $72 \mathrm{~h}$ of development was not significantly affected (ANOVA, $P=0.061$ ) by treatment. Values for eye diameter were $487 \pm 20 \mu \mathrm{m}$ for control $(n=34), 555 \pm 20 \mu \mathrm{m}$ for P-C saline $(n=15)$, and $512 \pm 10 \mu \mathrm{m}$ for the values of ZD7288 spanning the dose range from 7.5 to $30 \mu \mathrm{M}(n=$ 40). Similarly, no significant difference was found in a second indicator of developmental progress-cervical flexure-between the three groups $(P=0.126)$, with values of $125.5^{\circ} \pm$ $5.5^{\circ} \quad(n=13), \quad 130.5^{\circ} \pm 7.6^{\circ} \quad(n=13)$, and $127.8^{\circ} \pm 4.9^{\circ}$ ( $n=14$ ) in control, P-C saline-, and ZD7288-dosed embryos, respectively.

\section{Body Mass Effects of Chronic ZD7288 Treatment}

Mass was used an indicator of growth, independent of development, which was assessed as described above with eye diameter and cervical flexure. Wet mass of the control $(14.1 \pm$ $0.9 \mathrm{mg})$ and P-C saline-treated $(14.8 \pm 0.8 \mathrm{mg})$ embryos was not significantly different $(P=0.131)$; consequently, these populations were grouped together in subsequent analyses. Wet masses of embryos dosed with $7.5,10,15,20$, and $30 \mu \mathrm{M}$ of ZD7288 were also not significantly different $(P=0.053)$ from each other (fig. 11A). Collectively, wet mass $(11.6 \pm 0.8 \mathrm{mg})$ of the pooled ZD7288 treatment group was $22 \%$ smaller than the P-C saline-treated group $(14.8 \pm 0.5 \mathrm{mg} ; \mathrm{P}<0.001)$.

Dry mass of the control $(1.2 \pm 0.1 \mathrm{mg})$ and P-C salinetreated $(1.0 \pm 0.3 \mathrm{mg})$ embryos was not significantly different ( $P=0.335)$; consequently, these populations were grouped together in subsequent analyses. Dry masses of embryos dosed with $7.5,10,15,20$, and $30 \mu \mathrm{M}$ of $\mathrm{ZD} 7288$ were also not significantly different from each other $(P=0.175$; fig. $11 B)$. Collectively, dry mass $(1.2 \pm 0.4 \mathrm{mg})$ of the pooled ZD7288 treatment group was $33 \%$ smaller than the control group $(0.8 \pm 0.04 \mathrm{mg} ; P<0.001)$, mirroring the changes described for wet mass.

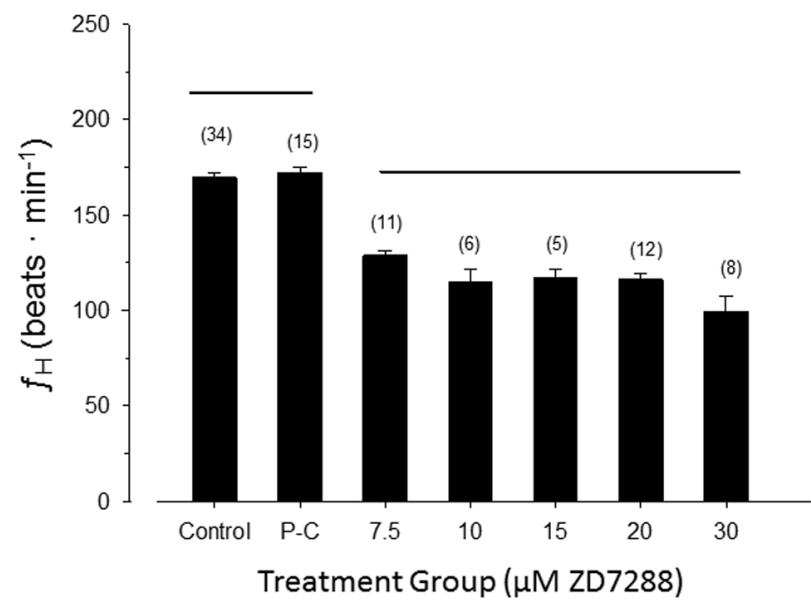

Figure 8. Heart rate at $72 \mathrm{~h}$ of development following $24 \mathrm{~h}$ of chronic ZD7288 dosing. Horizontal lines indicate statistically identical groups. Means \pm 1 SE are plotted; $n$ values are in parentheses. P-C, PannettCompton. 

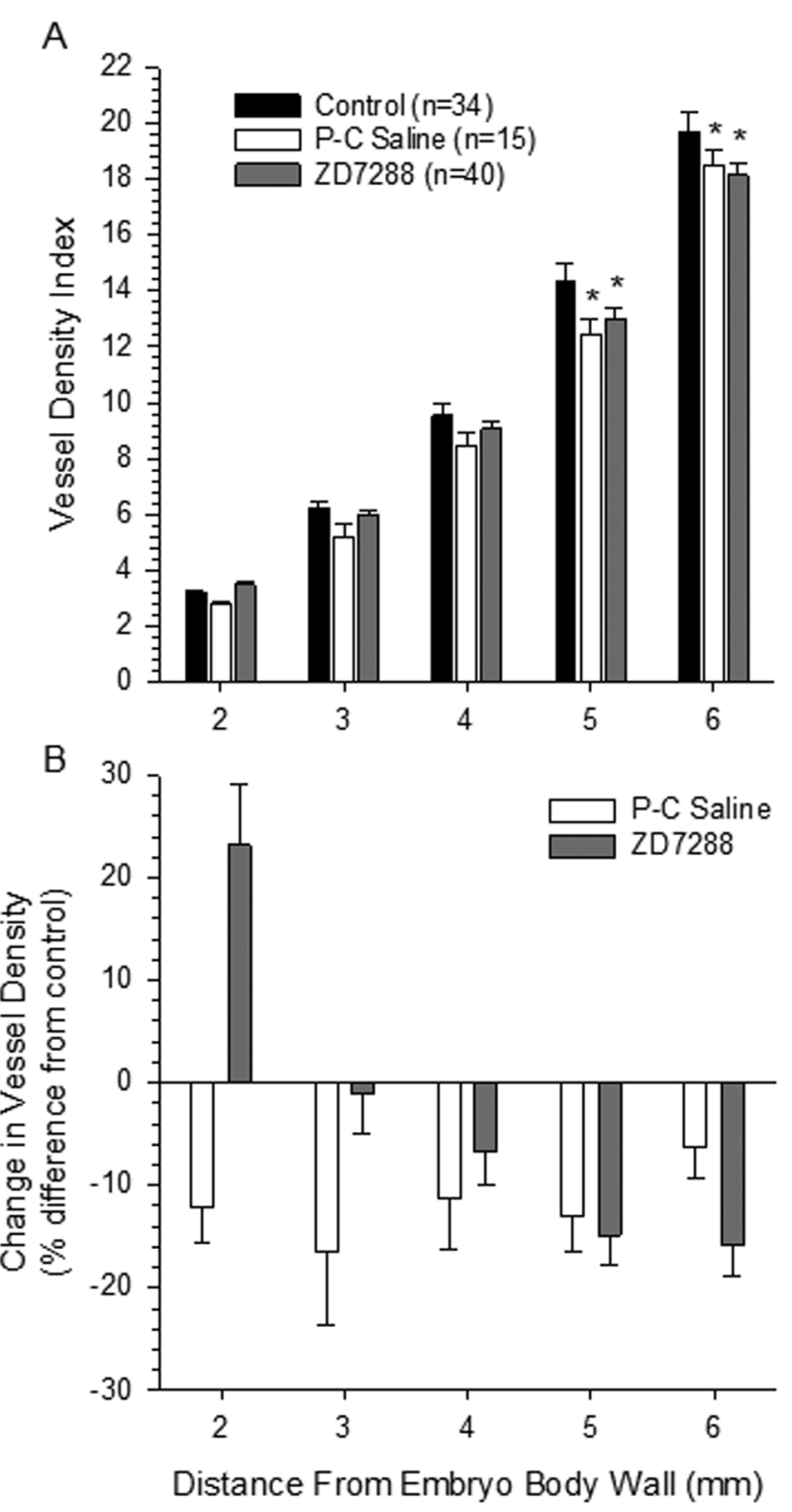

Figure 9. Chorioallantoic membrane (CAM) vessel density index at 72 $\mathrm{h}$ of development following $24 \mathrm{~h}$ of chronic ZD7288 dosing. CAM vessel density index is expressed in absolute terms $(A)$ and as a percent difference from control $(B)$. Asterisks indicate groups that are significantly different from control. Data are presented as means $\pm 1 \mathrm{SE} ; n$ values for each population are in parentheses. P-C, Pannett-Compton.

\section{Discussion}

\section{Critique of ZD7288 and Its Application}

ZD7288, which blocks the $I_{\mathrm{f}}$ cardiac pacemaker channels in the heart pacemaker, is widely regarded as a pure bradycardic drug that reduces $f_{\mathrm{H}}$ without producing inotropic changes in heart performance (e.g., Yusuf and Camm 2003; Luo et al. 2006). Acute dosing of ZD7288 at 30-, 250-, and 500- $\mu \mathrm{M}$ concentrations caused a bradycardia that was dependent on dose and time (fig. 7). The lowest concentration of $30 \mu \mathrm{M}$ ZD7288 caused a significant decrease in $f_{\mathrm{H}}$, but recovery occurred much more quickly than with the higher concentrations. Acute dosing at $72 \mathrm{~h}$ of development with 250 or $500 \mu \mathrm{M}$ ZD7288 caused the same degree of bradycardia (fig. 7). Similarly, the bradycardia induced by chronic ZD7288 measured at $72 \mathrm{~h}$ of development was both large and independent of dose above $7.5 \mu \mathrm{M}$ (fig. 8). There was no dose response found, suggesting that even the lowest concentration of ZD7288 saturates all the $I_{\mathrm{f}}$ cardiac pacemaker channels in the heart. In isolated mouse hearts, even smaller doses of ZD7288 $(1 \mu \mathrm{M})$ were sufficient to produce stable and profound bradycardia (67\% heart rate decrease) within 10 min of drug exposure, with a similar saturation effect (i.e., no further increase in heart rate) of ZD7288 at the higher concentrations (Stieber et al. 2003).

Heart rate of control embryos and embryos receiving chronic application of P-C saline were not significantly different (fig. 8 ). This indicates that the bradycardic effects caused by the application of ZD7288 were strictly pharmacological and not the result of the physical application of the vehicle by which the drug was administered, that is, P-C saline.

The highest concentration of $30 \mu \mathrm{M}$ ZD7288 used in our chronic experiments (which did not produce a significantly greater bradycardia than the far lower concentrations) nonetheless proved to be a lethal dose to $\sim 50 \%$ of the embryos. Additional studies to determine the effects of ZD7288 in older chicken embryos would help characterize this drug for potential use in future cardiovascular studies.

\section{Bradycardia, Pulse Pressure, and Angiogenesis}

Bradycardia and the associated increased diastolic duration increases pulse pressure in the heart and central arterial vessels in the chicken embryo (Buschmann et al. 2010). Increased pulse pressure in turn will create greater blood vessel wall distension (circumferential tension), increasing the degree of mechanical distension on the endothelial cells lining the blood vessels and potentially stimulating angiogenesis through the paracrine effects of VEGF (Groenendijk et al. 2007). In 3-d-old chicken embryos, the central aorta exhibits very modest cyclic stretch: only an approximately 3\% diameter excursion between systole to diastole (Buschmann et al. 2010). Vessels more peripheral to the aorta showed no measurable rhythmic distension associated with the cardiac cycle (Buschmann et al. 2010). These observations of low vascular compliance in the early chicken embryo, which would create little to no windkessel effect to maintain a diastolic runoff of blood flow, are compatible with the measurements of the current study, showing central aortic blood velocity falling sharply to 0 in early diastole (fig. 4).

The pulsatile blood flow of the early embryonic arterial system (fig. 4) is likely to create significant and variable shear stress along the walls of the developing vascular system. Moreover, the long-standing predictions that wall shear stress is constant through the vascular tree has been replaced with measurements showing both high regional variability as well as highest shear stress in the proximal vasculature, where total cross-sectional diameters are lowest and blood velocity is highest (see Stroev et al. 2007; Reneman and Hoeks 2008). Yet in 

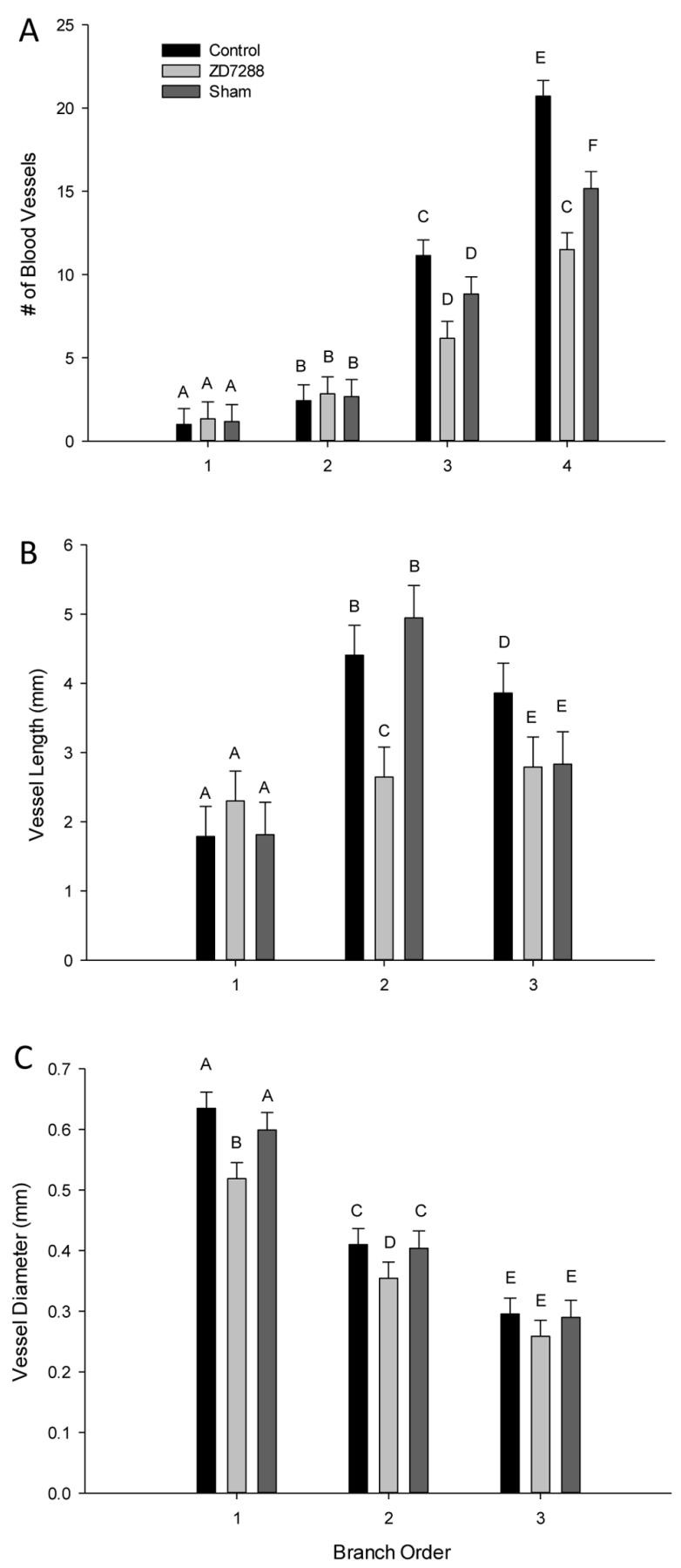

Figure 10. Effect of vessel branch order (1-3) on number of vessels $(A)$, vessel length $(B)$, and vessel diameter $(C)$ in control, Pannett-Compton (P-C) saline-treated, and ZD7288-treated embryos at $72 \mathrm{~h}$ of development. Letters indicate significance between branch orders and treatment groups (two-way ANOVA, Tukey pairwise comparison). Data are presented as means \pm SE; $n=7$ for each treatment group.

our experiments, induced bradycardia and the attendant changes in blood flow (and associated shear stress) and pulse pressure (and associated mechanical wall distention) did not stimulate angiogenesis (as quantified by the CAM vessel density index) in vessels falling within the range of $200-600 \mu \mathrm{m}$ from the embryo's umbilical stock (fig. 9), the very region predicted to have the highest shear stresses. In fact, chronic bradycardia and the attendant decrease in CO actually produced a small but significant decrease in CAM vessel density index in the most peripheral vasculature (500-600 $\mu \mathrm{m}$ from the embryo) when compared with the control and $\mathrm{P}-\mathrm{C}$ saline populations experiencing higher heart rates. A viable explanation could be that decreased heart rate and $\mathrm{CO}$ compromises perfusion and the bulk transport of materials necessary for angiogenesis, especially in the CAM periphery. The effects of ZD7288 were pronounced in the periphery of the CAM most likely as a cause of timing of drug application during development. The ZD7288 infusion began at $48 \mathrm{~h}$ of development after the initial vessel branch order had been formed. The effect of ZD7288 was seen in the outer branch orders in the smaller vessels that experienced angiogenesis during the experimental 24-h treatment.

The number of blood vessels increases with each increase in branch order as branch order increases in control embryos, as expected for a normal progression of angiogenesis in the CAM of control embryo. However, the embryos treated both with P-C saline and with ZD7288 exhibited a decrease in the number of blood vessels per branch order, indicating possible reduction in CAM vessel proliferation (fig. 10). ZD7288-treated embryos experiencing significant bradycardia had much shorter vessels in the outer branch order compared with control embryos, and while their vessel diameter was also smaller, the effect was not as pronounced. These data further indicate that bradycardia, the associated changes in blood flow and pulse pressure, and the anticipated changes in shear stress on the growing vessel walls do not stimulate angiogenesis at this stage of development, contrary to our initial hypothesis. Vessel diameter in the highest branch order was unaltered, showing additionally that a decreased $f_{\mathrm{H}}$ does not alter the size of the actual blood vessel formed but alters the process of normal angiogenesis, as represented by vessel number and length. These findings suggest several new avenues for research, including, for example, investigation of the ontogeny per se of the endothelial response to shear and circumferential stresses.

\section{Saline Application and Angiogenesis}

Unexpectedly, the mere topical application of $\mathrm{P}-\mathrm{C}$ saline produced a small but significant decrease in CAM vessel density index in the most peripheral vessels examined (fig. 9). The most parsimonious explanation is that chronic $\mathrm{P}-\mathrm{C}$ saline application to the embryo's surface washed out molecules important to normal CAM vessel development. A very wide range of proteins (e.g., transcription factors) and other molecules are important to angiogenesis (e.g., Chang and Hla 2011; GianniBarrera et al. 2011) and could be disturbed by extracellular pools of $\mathrm{P}-\mathrm{C}$ saline and the resulting concentration imbalances produced by saline moving across the surface of the growing CAM. Since the application of both P-C saline and ZD7288 decreased vessel density in the more peripheral regions of the CAM, this suggests that there may be a specific microenviron- 


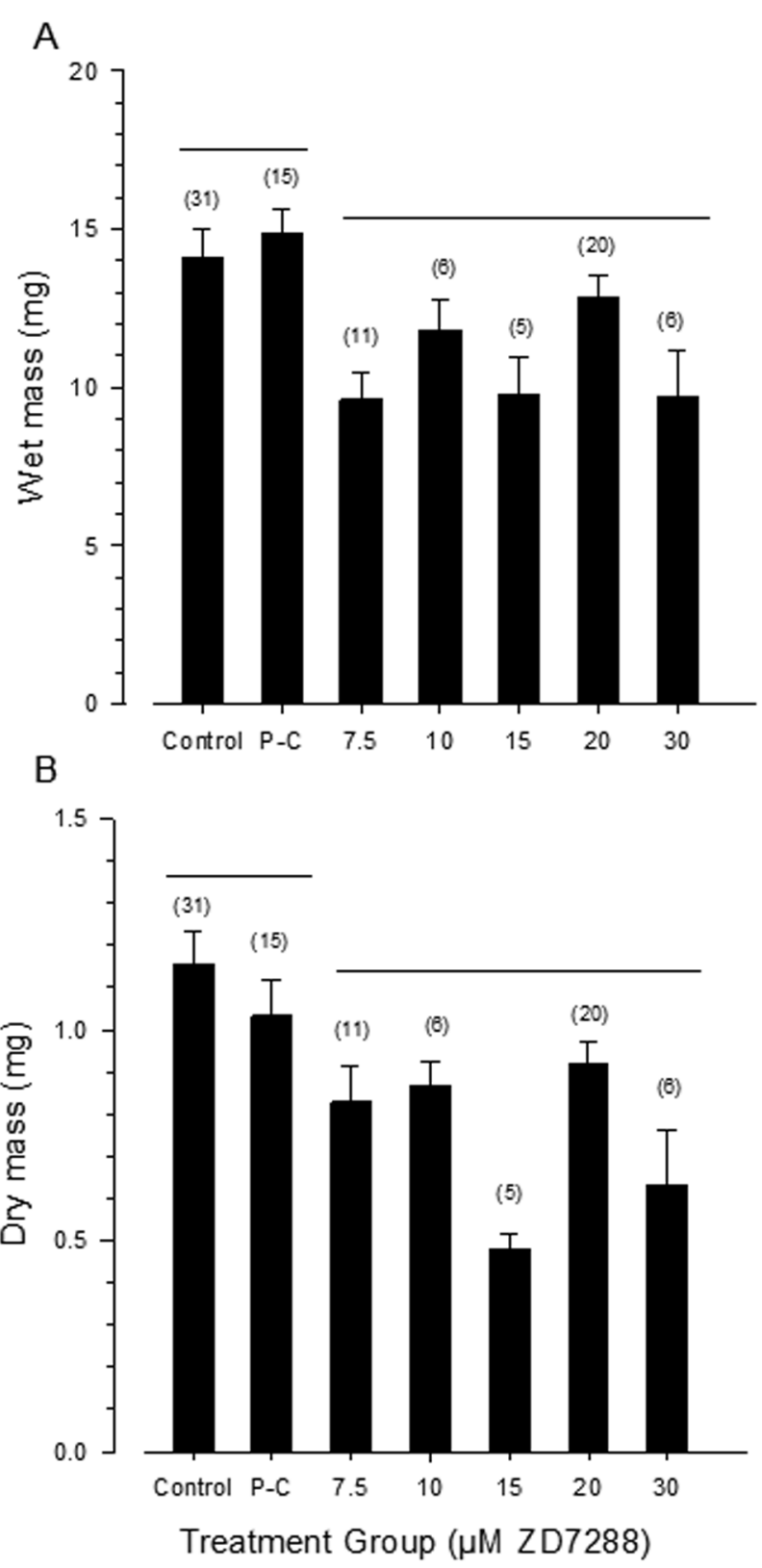

Figure 11. Wet mass $(A)$ and dry mass $(B)$ at $72 \mathrm{~h}$ of development following $24 \mathrm{~h}$ of chronic ZD7288 dosing at $48 \mathrm{~h}$ of development. Data are presented as means $\pm 1 \mathrm{SE} ; n$ values are in parentheses. Horizontal lines indicate groups that are statistically identical. P-C, PannettCompton.

mental balance that maintains normal CAM blood vessel development, at least in the peripheral if not more central regions of the CAM. The washout effect of saline-treated embryos was mainly seen at the periphery of the CAM, most likely because the initial branch orders were formed before treatment and the smaller vessels of the outer branch orders showed the effect that the washout of angiogenic factors had on decreasing vessel development. P-C saline was the vehicle for ZD7288. It is possible that this washing away of necessary angiogenetic factors- rather than a bradycardic effect of ZD7288 by itself-could have affected the development of vessels in the ZD7288-treated embryos as well.

\section{Influence of Cardiac Output on Development and Growth Rates}

Eye diameter is a standard method of tracking the rate of embryo development in the chicken embryo, not only because eye diameter is easy to visualize but also because the eyes show large, linear increase in size during 72-120 h (Romanoff 1960). Similarly, decreasing cervical flexure is taken as an indication of increasing embryonic development (Männer et al. 1995). There was no significant difference in either eye diameter or cervical flexure between any of the chronically treated groups, indicating that development rate (as distinct from growth) of the embryos was not altered by chronic bradycardia. Reduced $\mathrm{CO}$ induced by partial ligation of the ventricular outflow tract had no effect on eye diameter or cervical flexure in day 3-4 chicken embryos (Burggren et al. 2004). In this study, pharmacologically induced bradycardia was not compensated for by an increase in stroke volume (fig. 5), and so CO declined as a result of ZD7288 treatment at $56 \mathrm{~h}$. Other studies on early chicken embryos have similarly shown that $f_{\mathrm{H}}$ is a strong indicator of CO. For example, Bowers et al. (1996), examining $\sim 84$-h embryos, demonstrated that CO is linearly related to $f_{\mathrm{H}}$ (and preload) while end-stroke volume is linearly related to diastolic volume. Consequently, given the strong evidence for the fact that bradycardia decreased CO in embryos both slightly younger $(56 \mathrm{~h})$ as well as slightly older $(84 \mathrm{~h})$, we assume here that embryos acutely and chronically treated with ZD7288 and measured at $72 \mathrm{~h}$ (i.e., bracketed by the published data cited above) similarly experienced decreased CO directly as a result of bradycardia. We interpret from our data, then, that normal rates of development (evident from no disturbance to normal rates of eye development and cervical flexure) can continue, despite the abnormally low CO produced by a ZD7288-induced bradycardia. Expressed differently, normal progression of the embryo through successive early developmental stages is not dependent on normal levels of CO.

Does experimentally reduced CO directly influence growth (evident from embryo mass through cell division or hypertrophy, for example) as distinct from development rate measured by eye diameter and cervical flexure? Indeed, chronic bradycardia and the attendant reduction in CO significantly decreased both wet and dry body mass (fig. 11). Thus, experimentally reduced $\mathrm{CO}$ slowed absolute growth (measured by reduced body mass) but not development per se, as measured by eye diameter and cervical flexure, aligning with earlier experimental findings of Burggren et al. (2004).

\section{Conclusion}

Collectively, our data suggest that bradycardia at $48-72 \mathrm{~h}$ of development does not produce the hypothesized stimulation of angiogenesis, actually having the opposite effect in the most 
peripheral regions of the CAM vasculature. However, the accompanying reduced $\mathrm{CO}$ induced in this study has produced two interesting findings. First, the rate of development, expressed by the key developmental landmarks of eye diameter and cervical flexure, is not affected by reduced CO. Yet embryonic growth, assessed by wet and dry weight, is significantly reduced. These findings are important in light of the fact that the convective flow of blood is not required for continued oxygen consumption or development at these same developmental stages (Burggren et al. 2000; Burggren 2004). This study indicates that convective blood flow is indeed required for absolute growth of the embryo. Thus, it would appear that in early embryonic states, while diffusion of oxygen across the embryo body wall and of nutrients and waste products between various body compartments can maintain normal oxygen consumption, absolute growth is indeed dependent on adequate convective blood flow. This study presents the novel finding that different processes in the ontogeny of the early vertebrate embryo have differential sensitivities to perturbations in convective blood flow.

\section{Acknowledgments}

We thank Eric Willis, an undergraduate research student, for assistance in the collection of the number of vessels, length, and diameter data. This study was supported by National Science Foundation operating grant IOS-1025823 to W.B.

\section{Literature Cited}

Adams R.H. and A. Eichmann. 2010. Axon guidance molecules in vascular patterning. Cold Spring Harbor Perspect Biol 2: $\mathrm{a} 001875$.

Borer J.S. 2004. Drug insight: $I_{\mathrm{f}}$ inhibitors as specific heartrate-reducing agents. Nat Clin Pract Cardiovasc Med 1:103109.

Bowers P.N., J.P. Tinney, and B.B. Keller. 1996. Nitroprusside selectively reduces ventricular preload in the stage 21 chick embryo. Cardiovasc Res 31:E132-E138.

Burggren W.W. 2004. What is the purpose of the embryonic heart beat? or how facts can ultimately prevail over physiological dogma. Physiol Biochem Zool 77:333-345.

- 2013. Cardiovascular development and angiogenesis in the early vertebrate embryo. Cardiovasc Eng Tech (forthcoming).

Burggren W.W., S.J. Khorrami, A. Pinder, and T. Sun. 2004. Body, eye, and chorioallantoic vessel growth are not dependent on cardiac output level in day 3-4 chicken embryos. Am J Physiol Regul Integr Comp Physiol 287:R1399-R1406.

Burggren W.W., S.J. Warburton, and M.D. Slivkoff. 2000. Interruption of cardiac output does not affect short term growth and metabolic rate in day 3 and 4 chick embryos. J Exp Biol 203:3831-3838.

Buschmann I.R., A. Pries, B. Styp-Rekowska, P. Hillmeister, L. Loufrani, D. Henrion, Y. Shi, et al. 2010. Pulsatile shear and
Gja5 modulate arterial identity and remodeling events during flow-driven arteriogenesis. Development 137:2187-2196.

Carmeliet P., V. Ferreira, G. Breier, S. Pollefeyt, L. Kieckens, M. Gertsenstein, M. Fahrig, et al. 1996. Abnormal blood vessel development and lethality in embryos lacking a single VEGF allele. Nature 380:435-439.

Chang S.H. and T. Hla. 2011. Gene regulation by RNA binding proteins and microRNAs in angiogenesis. Trends Mol Med 17:650-658.

Corona T.B. and S.J. Warburton. 1999. Regional hypoxia elicits regional changes in chorioallantoic membrane vascular density in alligator but not chicken embryos. Comp Biochem Physiol 125:57-61.

DiFrancesco D. 2006. Funny channels in the control of cardiac rhythm and mode of action of selective blockers. Pharmacol Res 52:399-406.

Djonov V., M. Schmid, S.A. Tschanz, and P.H. Burri. 2000. Intussusceptive angiogenesis: its role in embryonic vascular network formation. Circ Res 86:286-292.

Dunnigan A., H. Norman, D.W. Benson Jr., and E.B. Clark. 1987. Effect of heart rate increase on dorsal aortic flow in the stage 24 chick embryo. Pediatr Res 22:442-444.

Egginton S. 2011. Physiological factors influencing capillary growth. Acta Physiol 202:225-239.

Ferrara N., K. Carver-Moore, H. Chen, M. Dowd, L. Lu, K.S. O'Shea, L. Powel-Braxton, K.J. Hillan, and M.W. Moore. 1996. Heterozygous embryonic lethality induced by targeted inactivation of the VEGF gene. Nature 380:439-442.

Gianni-Barrera R., M. Trani, S. Reginato, and A. Banfi. 2011. To sprout or to split? VEGF, notch and vascular morphogenesis. Biochem Soc Trans 39:1644-1648.

Gilbert S.F. 2006. Developmental biology. Sinauer, Sunderland, MA.

Groenendijk B.C., K. Van der Heiden, P.B. Hierck, and R.E. Poelmann. 2007. The role of shear stress on ET-1, KLF2, and NOS-3 expression in the developing cardiovascular system of chicken embryos in a venous ligation model. Physiology 22:380-389.

Grunz H. 1999. Amphibian embryos as a model system for organ engineering: in vitro induction and rescue of the heart anlage. Int J Dev Biol 43:361-364.

Hamamichi S. and H. Nishigori. 2001. Establishment of a chick embryo shell-less culture system and its use to observe change in behavior caused by nicotine and substances from cigarette smoke. Toxicol Lett 119:95-102.

Hamburger V. and H.L. Hamilton. 1951. A series of normal stages in the development of the chick embryo. J Morphol 88:49-92.

Heinke J., C. Patterson, and M. Moser. 2012. Life is a pattern: vascular assembly within the embryo. Front Biosci 4:22692288.

Höper J. and H. Jahn. 1995. Influence of environmental oxygen concentration on growth and vascular density of the area vasculosa in chick embryos. Int J Microcirc Clin Exp 15:186192. 
Hu N. and E.B. Clark. 1989. Hemodynamics of the stage 12 to stage 29 chick embryo. Circ Res 65:1665-1670.

Hu N., D.M. Connick, B.B. Keller, and E.B. Clark. 1991. Diastolic filling characteristics in the stage 12 to 27 chick embryo ventricle. Pediatr Res 29:334-337.

Isogai S., N.D. Lawson, S. Torrealday, M. Horiguchi, and B.M. Weinstein. 2003. Angiogenic network formation in the developing vertebrate trunk. Development 130:5281-5290.

Ji R.P., C.K.L. Phoon, O. Aristizabal, K.E. McGrath, J. Palis, and D.H. Turnbull. 2003. Onset of cardiac function during early mouse embryogenesis coincides with entry of primitive erythroblasts into the embryo proper. Circ Res 92:133-135.

Jones E.A., F. le Noble, and A. Eichmann. 2006. What determines blood vessel structure? genetic prespecification vs. hemodynamics. Physiology 21:388-395.

Kaunas R., H. Kang, and K.J. Bayless. 2011. Synergistic regulation of angiogenic sprouting by biochemical factors and wall shear stress. Cell Mol Bioeng 4:547-559.

Kirby M.L. 2007. Cardiac development. Oxford University Press, New York.

Knudsen T.B. and N.C. Kleinstreuer. 2011. Disruption of embryonic vascular development in predictive toxicology. Birth Defects Res C Embryo Today 93:312-23.

Lee H.S., J. Han, H.J. Bai, and K.W. Kim. 2009. Brain angiogenesis in developmental and pathological processes: regulation, molecular and cellular communication at the neurovascular interface. FEBS J 276:4622-4635.

le Noble F., C. Klein, A. Tintu, A. Pries, and I. Buschmann. 2008. Neural guidance molecules, tip cells, and mechanical factors in vascular development. Cardiovasc Res 78:232-241.

Luo L., L. Chang, S.M. Brown, H. Ao, D.H. Lee, E.S. Higuera, A.E. Dubin, and S.R. Chaplan. 2006. Role of peripheral hyperpolarization-activated cyclic nucleotide-modulated channel pacemaker channels in acute and chronic pain models in the rat. Neuroscience 144:1447-1485.

Männer J., W. Seild, and G. Stedng. 1995. Formation of the cervical flexure: an experimental study on chick embryos. Acta Anat 152:1-10.

McElhinney D.B., C.B. Benson, D.W. Brown, L.E. WilkinsHaug, A.C. Marshall, L. Zaccagnini, and W. Tworetzky. 2010. Cerebral blood flow characteristics and biometry in fetuses undergoing prenatal intervention for aortic stenosis with evolving hypoplastic left heart syndrome. Ultrasound Med Biol 36:29-37.

Mellish J.A.E., A.W. Pinder, and S.C. Smith. 1994. You've got to have heart. Or do you? Axolotl Newsl 23:34-38.

Michelfelder E., W. Polzin, and R. Hirsch. 2008. Hypoplastic left heart syndrome with intact atrial septum: utilization of a hybrid catheterization facility for cesarean section delivery and prompt neonatal intervention. Catheter Cardiovasc Interv 72:983-987.

Nowak-Sliwinska P., J.P. Ballini, G. Wagnieres, and H. Van den Bergh. 2009. Processing of fluorescence angiograms for the quantification of vascular effects induced by anti-angiogenic agents in the CAM model. Microvasc Res 79:21-28.
Pannett C.A. and A. Compton. 1924. The cultivation of tissues in orange juice. Lancet 206:381-384.

Pelster B. and W.W. Burggren. 1996. Disruption of hemoglobin oxygen transport does not impact oxygen-dependent physiological processes in developing embryos of zebrafish (Danio rerio). Circ Res 79:358-362.

Reneman R.S. and A.P. Hoeks. 2008. Wall shear stress as measured in vivo: consequences for the design of the arterial system. Med Biol Eng Comput 46:499-507.

Ribatti D., B. Nico, A. Vacca, L. Roncali, P. Burri, and V. Djonov. 2001. Chorioallantoic membrane capillary bed: a useful target for studying angiogenesis and anti-angiogenesis in vivo. Anat Rec 264:317-324.

Romanoff A.L. 1960. The avian embryo: structural and functional development. Macmillan, New York.

Rouse W., P.J. Stafford, and I.R. Johnson. 1994. The haemodynamic actions of ZENECA ZD7288, a novel sino-atrial node function modular, in the exercising beagle: a comparison with zatebradine and propranolol. Br J Pharmacol 113: 1071-1077.

Ruggiero M., D.P. Bottaro, G. Liguri, M. Gulisano, B. Peruzzi, and S. Pacini. 2004. 0.2 T magnetic field inhibits angiogenesis in chick embryo chorioallantoic membrane. Bioelectromagnetics 25:390-396.

Semerano L., G. Clavel, E. Assier, A. Denys, and M.C. Boissier. 2010. Blood vessels, a potential therapeutic target in rheumatoid arthritis? Joint Bone Spine 78:118-123.

Stern C.D. 1993. Avian embryos. Pp. 45-54 in C.D. Stern and P.W.H. Holland, eds. Essential developmental biology: a practical approach. IRL at Oxford University Press, Oxford.

Stern C.D. and D.R. Cannings. 1988. Gastrulation in birds, a model system for the study of animal morphogenesis. Experimentia 44:651-657.

Stieber J., S. Herrmann, S. Feil, J. Löster, R. Feil, M. Biel, F. Hofmann, and A. Ludwig. 2003. The hyperpolarizationactivated channel HCN4 is required for the generation of pacemaker action potentials in the embryonic heart. Proc Natl Acad Sci USA 100:15235-15240.

Strick D.M., R.L. Waycaster, J.P. Montani, W.J. Gay, and T.H. Adair. 1991. Morphometric measurements of chorioallantoic membrane vascularity: effects of hypoxia and hyperoxia. Am J Physiol H1385-H1389.

Stroev P.V., P.R. Hoskins, and W.J. Easson. 2007. Distribution of wall shear rate throughout the arterial tree: a case study. Atherosclerosis 191:276-280.

Territo P.R. and W.W. Burggren. 1998. Cardio-respiratory ontogeny during chronic carbon monoxide exposure in the clawed frog Xenopus laevis. J Exp Biol 201:1461-1472.

Wagner M. and M.A.Q. Siddiqui. 2007. Signal transduction in early heart development. I. Cardiogenic induction and heart tube formation. Exp Biol Med 232:852-865.

Wang X., C. Chen, L. Wang, D. Chen, W. Guang, and J. French. 2003. Conception, early pregnancy loss, and time to clinical pregnancy: a population-based prospective study. Fertil Steril 79:577-584.

Wilcox A.J., C.R. Weinberg, J.F. O’Conner, D.D. Baird, J.P. 
382 S. R. Branum, M. Yamada-Fisher, and W. Burggren

Schlattere, R.E. Canfield, E.G. Armstrong, and B.C. Nisula. Yusuf S. and A.J. Camm. 2003. Sinus tachyarrythmias and the 1988. Incidence of early loss of pregnancy. N Engl J Med specific bradycardic agents: a marriage made in heaven? J 319:189-194. Cardiovasc Pharmacol Ther 8:89-105. 
Copyright of Physiological \& Biochemical Zoology is the property of University of Chicago Press and its content may not be copied or emailed to multiple sites or posted to a listserv without the copyright holder's express written permission. However, users may print, download, or email articles for individual use. 